\title{
Análisis funcional del nicho ecológico de Syphrea sp. (Coleoptera: Chrysomelidae) asociado a Plukenetia volubilis L. (Euphorbiales: Euphorbiaceae) en la Amazonia peruana
}

\author{
Carlos D. Vecco ${ }^{1}$, Román Pinedo ${ }^{1}$, Miriam Fernández ${ }^{2}$ y Ronald D. Cave ${ }^{3}$
}

\begin{abstract}
Resumen. Se evaluó el comportamiento y la distribución de adultos de Syphrea sp. en plantas de Plukenetia volubilis L. (Euphorbiaceae) bajo un sistema de cultivo de alta densidad en una zona cercana a Tarapoto, Perú. Se examinaron, diariamente, cinco plantas al azar por bloque en tres horarios (8:00-9:00, 13:00-14:00 y 17:00-18:00), teniendo en cuenta el número de adultos de Syphrea sp. en 10 hojas (haz y envés) y 10 inflorescencias tomadas aleatoriamente en cada estrato (inferior, medio, y superior). Se realizó un análisis funcional considerando 45 dimensiones que expresan las interacciones de tipo estructural, trófico, y temporal. Se calculó la amplitud del nicho $\left(\mathrm{B}_{\mathrm{j}}\right)$ y determinó la dependencia de las poblacionales de Syphrea sp. con respecto a las dimensiones consideradas. Como resultado, se sugiere la especialización trófica y reproductiva de Syphrea sp. en hojas jóvenes e inmaduras, y quizás polen, en la parte externa de la planta, particularmente en el tercio superior. El comportamiento diurno del insecto y su tendencia a concentrarse al atardecer, podría sugerir que la alimentación es una actividad nocturna. Con respecto al aporte de elementos para la metodología del muestreo, se demostró la ventaja del uso de niveles estructurales referenciales (estratos por tercios) y se recomendó realizar las evaluaciones al atardecer.
\end{abstract}

Palabras clave: Amplitud del nicho, comportamiento, distribución en planta, muestreo, sacha inchik, .

Abstract. The behavior and within-plant distribution of adult Syphrea sp. was evaluated on Plukenetia volubilis L. (Euphorbiaceae) under a high density planting system near Tarapoto, Perú. Five randomly selected plants per block were observed daily during three 1-hour periods (8:00-9:00, 13:00-14:00 and 17:00-18:00), taking into account the number of adult Syphrea sp. on 10 leaves (upper and lower surfaces) and 10 inflorescences chosen randomly from each plant stratum (lower, middle and higher). A functional analysis considered 45 dimensions that express structural, trophic, and temporal interactions. Niche breadth $\left(\mathrm{B}_{\mathrm{j}}\right)$ was calculated and dependence of Syphrea sp. population levels with respect to the dimensions considered was determined. Adults of Syphrea sp. trophically and reproductively specialize on young and immature leaves, and perhaps pollen, on the external part of the plant, particularly the upper third. The diurnal behavior of Syphrea sp. is inferred and the beetle's tendency to aggregate at dusk suggests that feeding is a nocturnal activity. The advantage of using referential structural levels and evaluating populations at dusk in a sampling methodology are discussed.

Key words: Behavior, inca peanut, niche breadth, sampling, within-plant distribution.

\section{Introducción}

Plukenetia volubilis L. (Euphorbiales: Euphorbiaceae) es una planta amazónica de hábito trepador, y es comunmente llamada sacha inchik. Sus semillas procesadas son comestibles y tienen un alto contenido de aceites esenciales (Omega 3, 6 y 9), cuya extracción industrial ha generado una demanda inicial y la promoción de su cultivo comercial en el Perú. La implementación de monocultivos coincide con la presencia de artrópodos fitófagos que eventualmente pueden ocasionar pérdidas productivas, económicas, $\mathrm{y}$ ambientales. Syphrea sp. (Coleoptera: Chrysomelidae: Galerucinae), una plaga que se alimenta del follaje de $P$. volubilis, fue advertido a mediados de 2006 en poblaciones altas causando $>30 \%$ defoliación en la zona húmeda tropical de Pongo de Caynarachi (210 msnm) en la Región de San Martín, Perú. Sin embargo, tuvo menor presencia en campos en la zona seca tropical de Tarapoto $(275-425 \mathrm{msnm})$ distante

${ }^{1}$ Estudios Amazónicos, Jr. Saposoa 181, Tarapoto, Perú, carvec2@yahoo.es, ropire5@hotmail.com

2 Instituto de Investigaciones en Fruticultura Tropical- IIFT, Ave. 7 a \# e/. 32 y 30, Miramar Playa, La Habana, Cuba, miriamfernandez@iift.cu

${ }^{3}$ Indian River Research \& Education Center, University of Florida, 2199 South Rock Rd, Ft. Pierce, FL, EEUU, rdcave@ufl.edu 
sólo a $60 \mathrm{~km}$ de Pongo de Caynarachi. Tomando en cuenta el potencial de daño al cultivo, se inició una investigación para determinar algunas características asociadas al comportamiento y monitoreo de los adultos de Syphrea sp.

Syphrea Baly, 1876 es un género grande con más de 100 especies en el Nuevo Mundo (Scherer 1983). La taxonomía del grupo está en mal estado; algunas especies probablemente se deben transferir a otros géneros. La identificación de las especies es muy difícil, por lo tanto, no se pudo identificar la especie que ataca a $P$. volubilis en el área de Tarapoto, Perú.

La disposición espacial de los adultos de Syphrea sp. (vertical y por recurso) dentro de la planta de $P$. volubilis y su relación con la variación temporal diaria son los aspectos abordados por este trabajo, para comprender mejor su comportamiento. Los objetivos del estudio fueron: (i) determinar la amplitud del nicho ecológico ${ }^{4}$ de Syphrea sp.; (ii) definir las relaciones estructurales, tróficas, y temporales del comportamiento diurno de Syphrea sp. en la planta de $P$. volubilis; (iii) aportar elementos para el ajuste de un método de muestreo del insecto.

\section{Materiales y Métodos}

El trabajo se realizó durante julio y agosto de 2007 en una parcela cuadrada de $2,025 \mathrm{~m}^{2}$ en el Centro Pukalluichu en Tarapoto, Perú (18M0350392, UTM 9285320; $425 \mathrm{msnm})$. El cultivo de $P$. volubilis se instaló en agosto de 2006 bajo el sistema de mata adensada que consiste en la siembra directa de las semillas, sin tutores, y distanciamientos de $1 \mathrm{~m}$ entre plantas y $3 \mathrm{~m}$ entre hileras.

Se evaluó diariamente en tres horarios (8:00-9:00, 13:00-14:00 y 17:00-18:00) con dos evaluadores durante 10 días. Durante este período hubo condiciones climáticas normales para la estación: sin lluvias y cielo predominantemente despejado. La parcela fue dividida en cinco bloques de igual tamaño, cuatro periféricos y uno central. Se examinaron cinco plantas al azar en cada bloque. Utilizando un muestreo del tipo aleatorio estratificado, se registró el número de

\footnotetext{
${ }^{4}$ La definición conceptual de nicho ecológico en el presente trabajo es la teoría n- volumétrica o del hipervolumen propuesta por Hutchinson (1957).
}

adultos de Syphrea sp. en 10 hojas (haz y envés) y en 10 inflorescencias tomadas aleatoriamente en el estrato inferior, medio y superior de cada planta seleccionada (Vecco 2006). También se registró la localización del insecto con respecto a la superficie expuesta de la planta (externa e interna). La condición fenológica del follaje (hojas senescentes, maduras, jóvenes, e inmaduras) pasó a ser información secundaria, registrada sólo en presencia del insecto.

Para determinar el grado de dependencia de las magnitudes de poblaciones de adultos de Syphrea sp. con respecto al estrato, horario de evaluación $\mathrm{y}$ ubicación del insecto en las hojas o en las plantas, se utilizaron técnicas estadísticas paramétricas (análisis de varianza bifactorial basado en la distribución de $F$, la prueba de $t$, separación de medias de Duncan) y la técnica no paramétrica Chi-cuadrada $\left(X^{2}\right)$. Se proporcionó la desviación estándar de los promedios. Las relaciones entre las dimensiones del subnicho temporal (horarios de 8:00-9:00, 13:00-14:00 y 17:0018:00) con respecto a la población del insecto se determinaron con el análisis de correlación lineal de Pearson, estimándose la función de regresión más aproximada al comportamiento de los datos.

En el análisis funcional se consideraron las interacciones del tipo estructural, trófico y temporal como subnichos, y como nicho total al conjunto integrado de estas interacciones (Fontenla et al. 1987), clasificadas en 45 dimensiones definidas por las categorías evaluadas en el trabajo de campo (Cuadro 1). La medida de la Amplitud del Nicho $\left(\mathrm{B}_{\mathrm{j}}\right)$ se calculó por la relación $\mathrm{B}_{\mathrm{j}}=\left(\mathrm{S} . \Sigma \mathrm{p}_{\mathrm{ij}}{ }^{2}\right)^{-1}$, basada en el índice propuesto por Levins (1968), donde $\mathrm{p}_{\mathrm{ij}}$ es la proporción de la especie i encontrada en una unidad de un grupo de recursos/especie $\mathrm{j}$, y $\mathrm{S}$ es la cantidad total de dimensiones o recursos ( $\mathrm{S}$ total $=45)$.

\section{Resultados}

Las densidades de la población de adultos de Syphrea sp. indicaron una correlación al horario de evaluación $\left(X^{2}=153.4 ; \mathrm{gl}=2 ; P<0.001\right)$. La mayor población fue al atardecer (17:00-18:00), con $1.6 \pm 0.4$ individuos por planta, que difirió en forma altamente significativa de las evaluaciones matutinas (8:00-9:00) y del inicio de la tarde (13:00-14:00), con $1.1 \pm 0.4$ y $0.8 \pm 0.3$ individuos por planta, respectivamente (Fig. 1). 
Cuadro 1. Número total de adultos de Syphrea sp. por localización (estrato de la planta y superficie de la hoja, o inflorescencia) e inicio de la hora de evaluación en 25 plantas de Plukenetia volubilis, Tarapoto, Perú, julio agosto de 2007.

Localización Hora de evaluación

Estrato

\begin{tabular}{llll}
\hline $8: 00$ & $13: 00$ & $17: 00$
\end{tabular} Totales

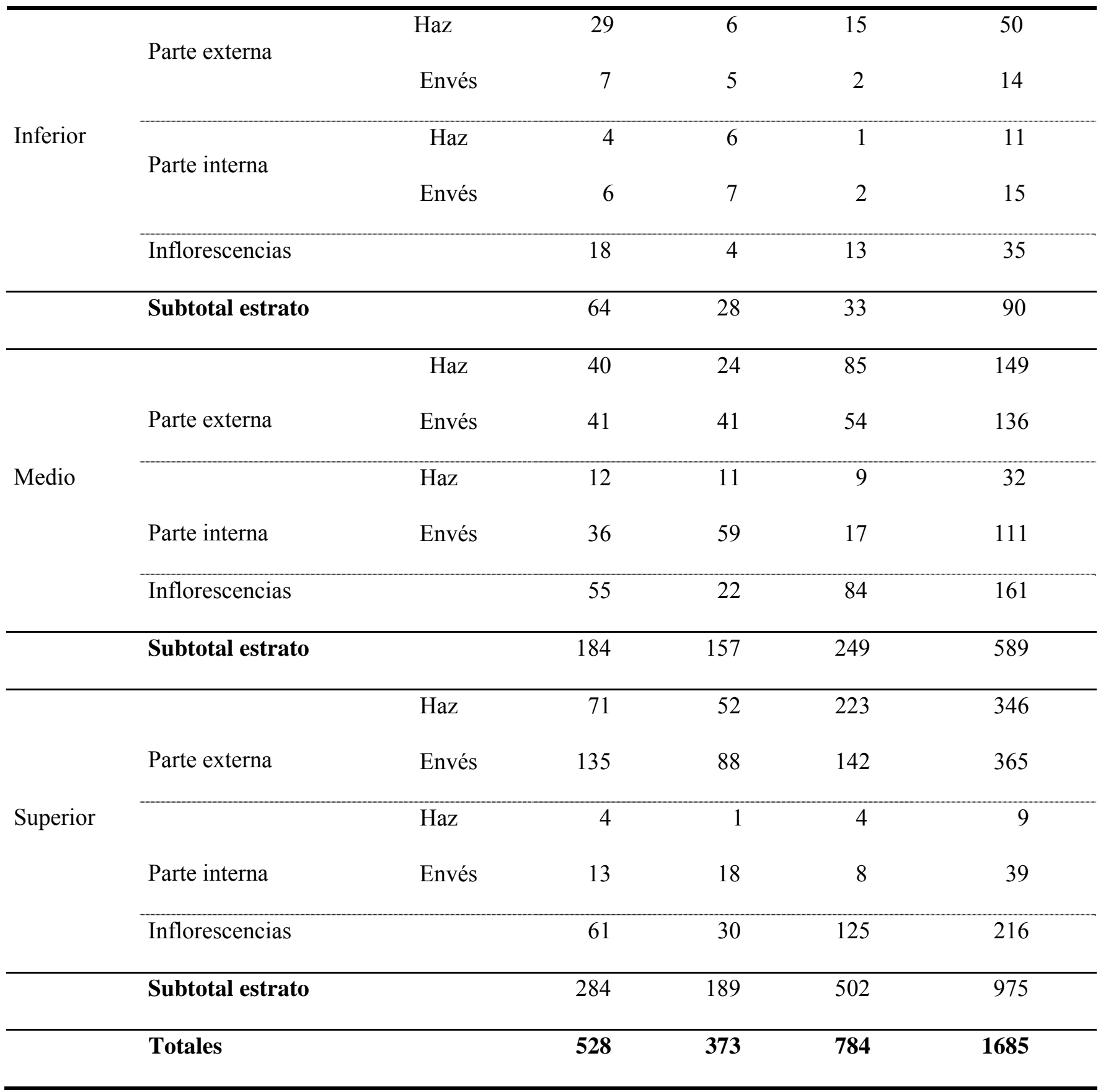




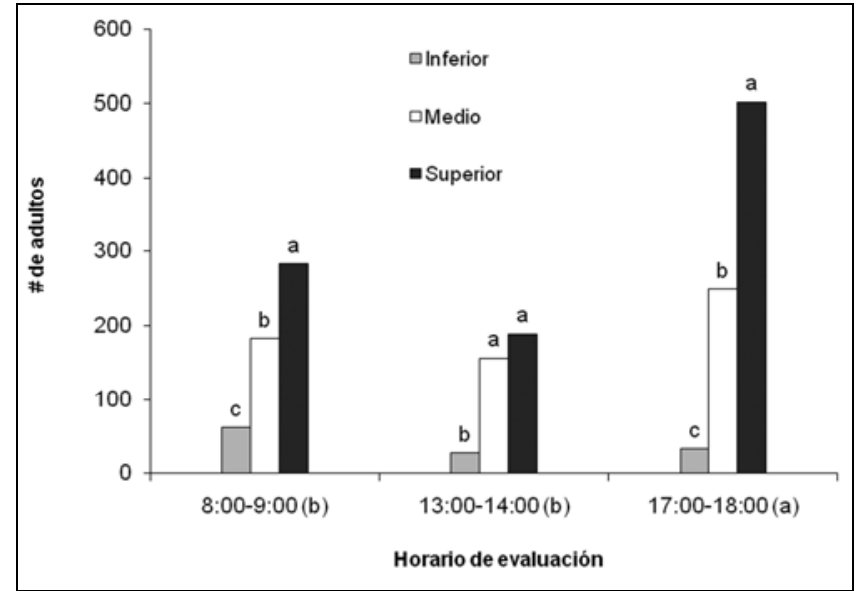

Figura 1. Población de adultos de Syphrea sp. en plantas de Plukenetia volubilis en relación con los estratos y el horario de evaluación. Barras del mismo horario $y$ horarios con letras iguales no son significativamente diferentes (Duncan, $P>0.01$ ).

También se demostró la correlación de la cantidad de adultos de Syphrea sp. con el subnicho estructural $\left(X^{2}=646.5 ;\right.$ g.l. $\left.=2 ; P<0.001\right)$. El estrato superior registró la mayor población con $0.7 \pm 0.4$ individuos por planta, seguido del estrato medio $(0.4 \pm$ 0.2 individuos por planta), y finalmente, del inferior $(0.1 \pm 0.1$ individuos por planta). Asimismo, se evidenció que esta disposición por estratos fue afectada por el horario de evaluación (interacción estrato por horario) en forma altamente significativa ( $F$ $=15.27$; g.1. $=4 / 171 ; P<0.001)$ (Fig. 1).

La ubicación de los adultos en la superficie de las hojas de $P$. volubilis fue altamente significativa con el horario de evaluación $(F=16.64 ;$ g.l. $=2 / 114$; $P<0.001)$. Mayor proporción de individuos se observó en el envés de las hojas en la mañana $(59.6 \%)$ y el inicio de la tarde $(68.6 \%)$, mientras que la situación se invirtió al atardecer (60.0\% en el haz) (Fig. 2).

En todos los horarios de evaluación, la parte externa de la planta registró el mayor número de adultos de Syphrea sp. con respecto a la parte interna $\left(X^{2}=557.8 ;\right.$ g.1. $\left.=1 ; P<0.001\right)$. Sin embargo, las proporciones variaron con el horario de evaluación $(F$ $=33.1 ;$ g.l. $=2 / 114 ; P<0.001)$, incrementándose la población de Syphrea sp. en las hojas internas de las plantas al inicio de la tarde (13:00-14:00) (Fig. 3).

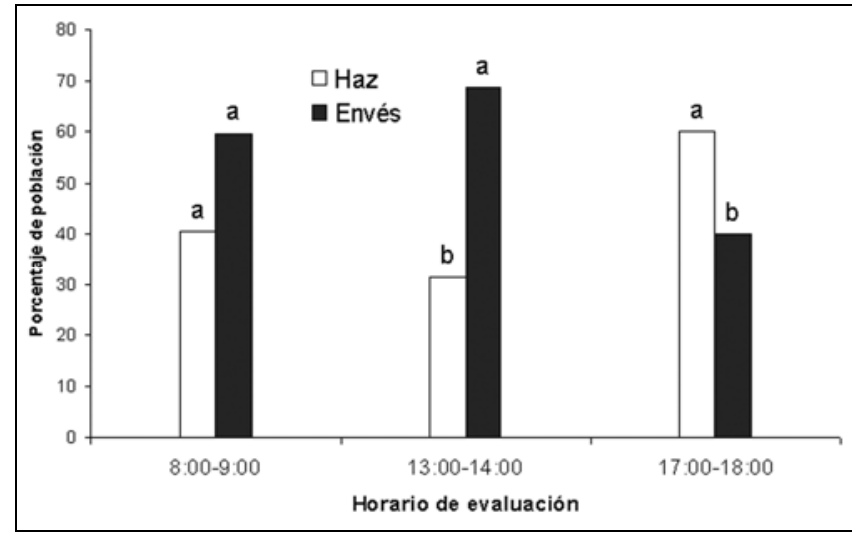

Figura 2. Porcentaje de la población de adultos de Syphrea sp. en Plukenetia volubilis en relación a su posición en la hoja y el horario de evaluación. Barras del mismo horario con letras iguales no son significativamente diferentes (prueba de $t, P>0.01$ ).

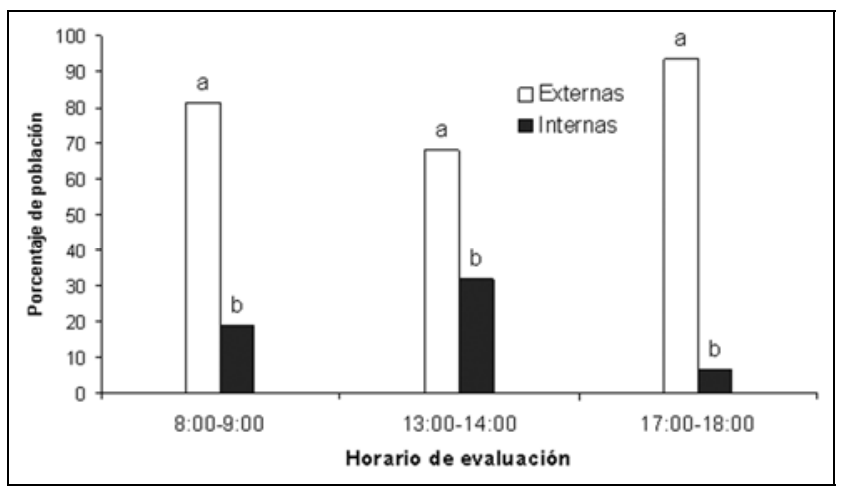

Figura 3. Porcentaje de la población de adultos de Syphrea sp. en plantas de Plukenetia volubilis en relación a su posición externa o interna en la planta y el horario de evaluación. Barras del mismo horario con letras iguales, no son significativamente diferentes (prueba de t, $P>0.01$ ).

La ocurrencia de adultos de Syphrea sp. en las hojas con diverso estado fenológico (senescentes, maduras, jóvenes, e inmaduras) mostró una alta dependencia con respecto al horario de evaluación $\left(X^{2}\right.$ $=26.65 ;$ g.l. $=6 ; P=0.02)$ y también hacia el estrato $\left(X^{2}=235.2\right.$; g.l. $\left.=6 ; \mathrm{P}<0.001\right)$. En el horario de 13:0014:00 la abundancia relativa de Syphrea sp. en hojas maduras fue más alta (69.5\%), mientras que para las 
hojas jóvenes e inmaduras se elevó en las horas de la mañana y el atardecer (43.8 y $45.7 \%$, respectivamente) (Fig. 4). Asimismo, el estrato superior presentó una mayor abundancia relativa de Syphrea sp. en las hojas jóvenes e inmaduras juntas (58.8\%), mientras que las hojas maduras fueron las más ocupadas en el estrato inferior (58.0\%) y medio de la planta (83.1\%) (Fig. 5). Del total de plantas evaluadas durante el periodo, un promedio de 13 y $28 \%$ no contaron con hojas jóvenes ni inmaduras, respectivamente.

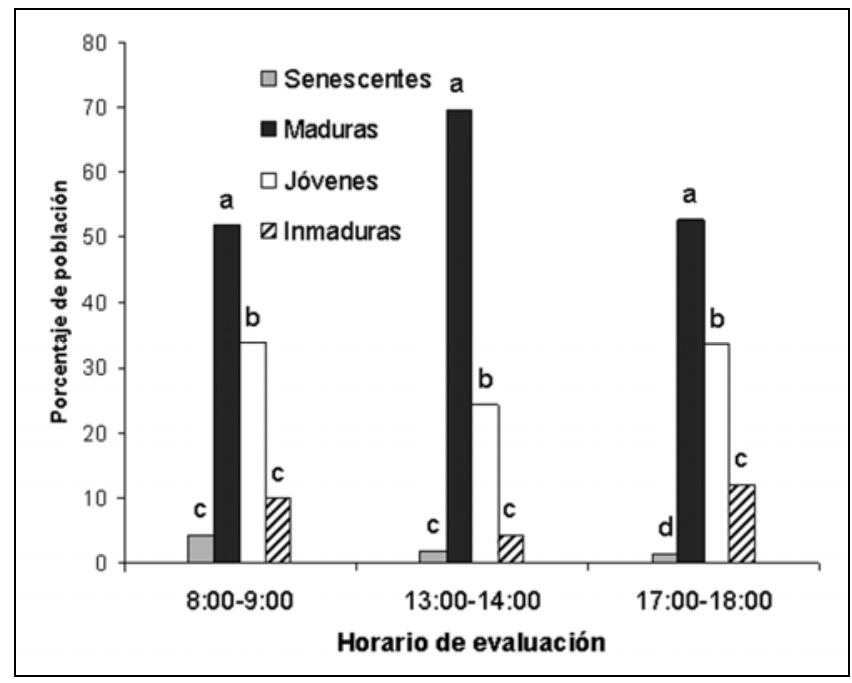

Figura 4. Porcentaje de la población de adultos de Syphrea sp. en hojas de Plukenetia volubilis de acuerdo a su estado fenológico por cada horario de evaluación. Barras del mismo horario con letras iguales no son significativamente diferentes (Duncan, $P>0.01$, datos transformados al $\log [X+10])$.

Los horarios 8:00-9:00 y 13:00-14:00 presentaron una correlación lineal altamente significativa $(r=0.85$; g.l. $=18 ; P<0.001)$ y una recta de regresión igualmente significativa $(F=45.34 ;$ g.l. $=1 / 18 ; P<0.001)$ de la forma: $Y=7.7+1.0 X$, donde $Y$ es la población estimada para el horario 8:00-9:00, y $X$ es la población del horario 13:00-14:00 (Fig. 6). Las comparaciones de los datos obtenidos en los horarios 8:00-9:00 y $17: 00-18: 00$ y en 13:00-14:00 y 17:00-18:00 no mostraron correlaciones lineales significativas. Sin embargo, al eliminarse tres pares de datos distorsionantes del total de 20 evaluaciones, la relación de los horarios 8:00-9:00 y 17:00-18:00 alcanzó alta significatividad $(r=0.70 ;$ g.l. $=15 ; \mathrm{P}<0.01)$ y se ajustó a la función lineal $Y=16.8+0.97 X(F=14.64$; g.l. $=$ $1 / 15 ; P=0.0017$ ) (Fig. 6). La relación de los horarios 13:00-14:00 y 17:00-18:00 no ajustó significativamente a la función lineal $Y=28.1+0.7 X$ $(F=4.13 ;$ g.1. $=1 / 15 ; P=0.06)$ (Fig. 6).

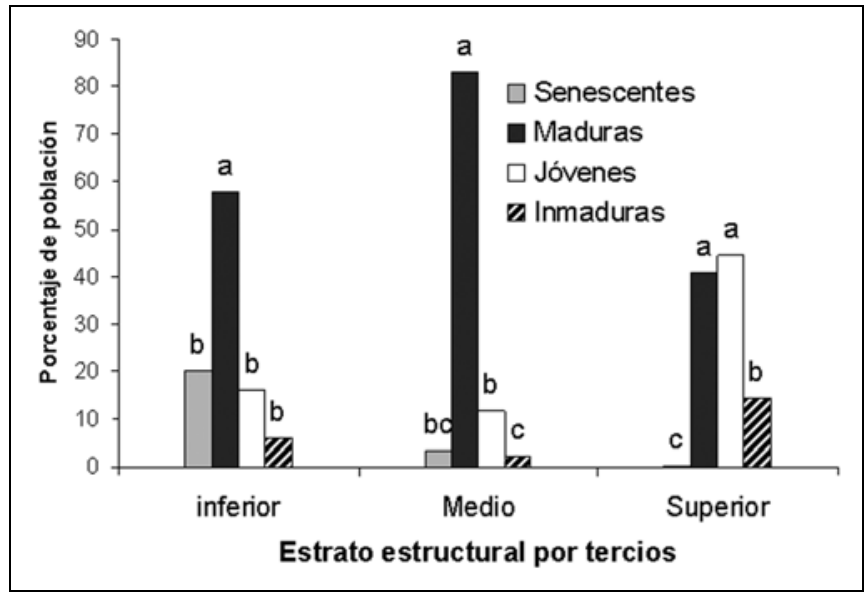

Figura 5. Proporción de la población de adultos de Syphrea sp. en hojas de Plukenetia volubilis de acuerdo a su estado fenológico por estratos estructurales. Barras del mismo horario con letras iguales no son significativamente diferentes (Duncan, $P>0.01$, datos transformados al $\log [X+10])$.

La amplitud total del nicho ecológico de los adultos de Syphrea sp. para las dimensiones consideradas fue estimada por el valor $\mathrm{Bj}=0.4004$. La amplitud varió en cada dimensión del subnicho temporal: en la mañana (8:00-9:00) fue 0.5175; pasado el mediodía (13:00-14:00) el valor fue 0.5122; al atardecer (17:00-18:00) fue 0.3957. Los adultos de Syphrea sp. presentaron mayor amplitud en el estrato medio $(\mathrm{Bj}=0.7315)$ con respecto a los estratos inferior y superior (con valores $\mathrm{Bj}$ de 0.5647 y 0.5088 , respectivamente). 

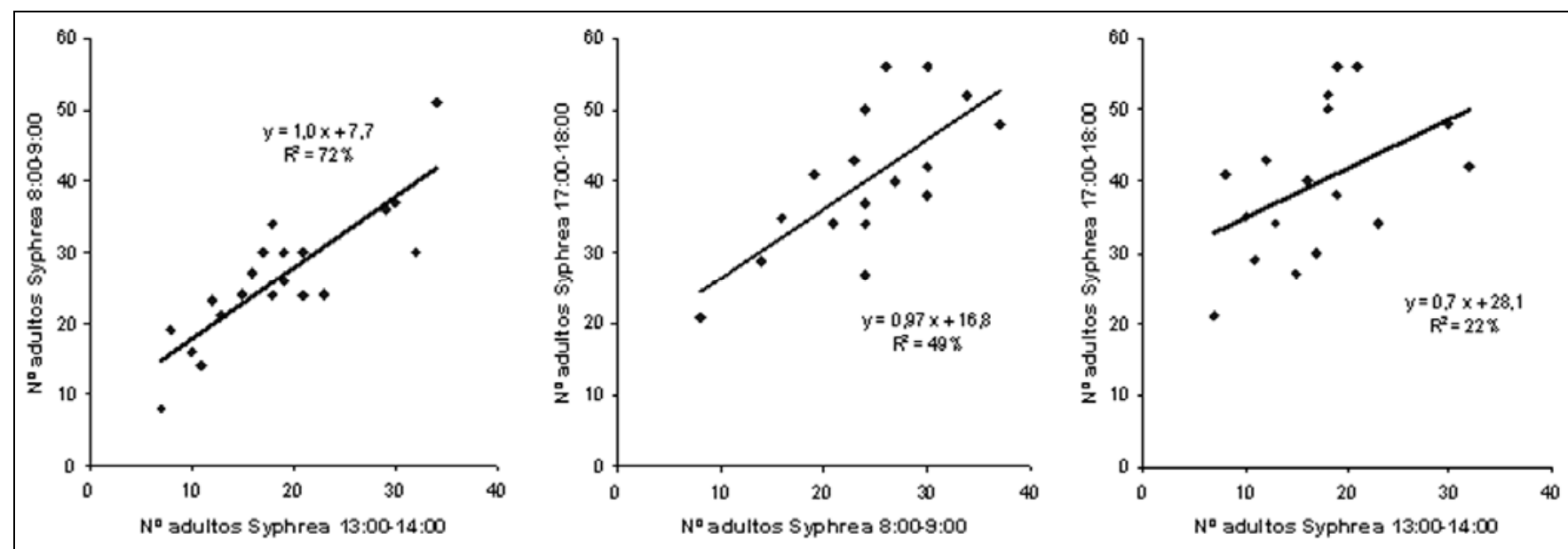

Figura 6. Correlaciónes lineales de las poblaciones de adultos de Syphrea sp. en 25 plantas de Plukenetia volubilis entre tres horarios de muestreo.

\section{Discusión}

Los resultados constituyen evidencias valiosas para inferir sobre la localización y movilidad diurna de los adultos de Syphrea sp. en la planta de P. volubilis. Los valores de la amplitud del nicho ecológico evidencian una distribución temporal definida de Syphrea sp. en ciertos recursos, la cual sugiere un comportamiento asociado a las condiciones ambientales del microhabitat y/o un significado principalmente trófico y posiblemente reproductivo. La observación directa de adultos alimentándose y apareándose exclusivamente al atardecer, parece explicar la reducción de la amplitud del nicho ecológico en este horario. Esta observación coincide con la preferencia alimenticia de los adultos de Syphrea sp. sobre hojas jóvenes e inmaduras, y quizás polen. Estos recursos se encuentran en mayor abundancia en la parte externa de la planta, particularmente en el tercio superior. Al atardecer, cuando las condiciones ambientales (temperatura y radiación solar) parecen ser más favorables para la actividad de Syphrea sp., la movilización y concentración de los individuos en torno a los recursos alimenticios del estrato superior pudieron favorecer también los encuentros sexuales.

El mayor valor de la amplitud del nicho ecológico para la mañana (8:00-9:00) indica que los insectos estuvieron más dispersos o más uniformemente distribuidos dentro de la planta. Después de esta hora, los adultos de Syphrea sp. tienden a refugiarse en el envés de las hojas, en la parte interna de la planta, o posiblemente en otras partes no muestreadas o poco accesibles para el observador (como hojarasca, hojas en contacto con el suelo u hospedantes alternos), como una respuesta al incremento de la temperatura ambiental, la cual se aproxima al máximo durante el inicio de la tarde (13:00-14:00). Esto podría explicar la respuesta sistemática del descenso poblacional (error de muestreo), el incremento percibido de la población de Syphrea sp. en las partes internas, y la reducción (aunque pequeña) de la amplitud del nicho ecológico en el horario de 13:00-14:00.

Se puede considerar la tendencia del comportamiento diurno de los adultos de Syphrea sp. para inferir los aspectos relacionados a su comportamiento nocturno. A pesar de que las hojas jóvenes e inmaduras de $P$. volubilis constituyen recursos tróficos indiscutibles, se pudo observar una importante proporción de individuos aún fuera de este recurso al atardecer, lo cual puede asociarse a la escasez de hojas jóvenes e inmaduras causada por la estación seca sobre la fenología del cultivo. Sin embargo, una consideración alternativa es que la lógica del comportamiento diurno de Syphrea sp. y su tendencia a concentrarse hacia el atardecer puede sugerir que la alimentación es una actividad frecuentemente nocturna, y que la amplitud del nicho ecológico podría descender aún más en la noche, 
evidenciando mayor especificidad de estos insectos.

Otras implicancias de los resultados son las que se podrían derivar de la validación del método de muestreo. Con respecto a la estratificación del cultivo, se demostró la ventaja del uso de niveles estructurales referenciales (estratos por tercios) con respecto a dimensiones estrictamente tróficas (hojas por edad), debido a que la disponibilidad de cada recurso varía con la fenología del cultivo, o porque la ubicación de las poblaciones durante el día son estados transicionales que no necesariamente se relacionan a la manifestación de la función trófica.

El muestreo al atardecer (17:00-18:00) parece brindar ciertas ventajas porque los insectos son más conspicuos y están concentrados en pocos recursos, siempre y cuando el tamaño requerido de muestra no influya limitando la duración del muestreo. En la presente experiencia, la duración del muestreo para evaluar 25 plantas fue de $56 \pm 6$ minutos en la tarde, ligeramente menor al promedio general de 66 minutos. Las evaluaciones al atardecer registran además el mayor número de adultos, lo cual sería más objetivo cuando se quiera relacionar los índices poblacionales del insecto con su capacidad de daño económico.

El uso de las funciones lineales que describen las relaciones entre poblaciones registradas para diferentes horarios, con cierta probabilidad de error, podría ser útil para ajustar los resultados de las evaluaciones que por diversas razones no puedan realizarse en el momento más oportuno. No obstante, estas funciones pueden depender fuertemente de las características fenotípicas particulares de la parcela, dependiendo del diseño del cultivo, estado fenológico, genotipo, y condiciones ambientales.

Las consideraciones sobre el comportamiento de los adultos de Syphrea sp. dentro de la planta de $P$. volubilis deben ser incorporadas al diseño de la metodología de muestreo del insecto y en los registros posteriores, para determinar los coeficientes de agregación (disposición espacial en la parcela) y el tamaño de muestra en función de los valores estadísticos de población.

\section{Literatura Citada}

Fontenla, J.L., R. Rodríguez, y M. Suri. 1987. Estructura y organización de dos comunidades de Coccoidea en dos cultivares de cítricos. Instituto de Ecología y Sistemática, La Habana, Cuba. Reporte de Investigación $\mathrm{n}^{\circ} 45.28 \mathrm{p}$.

Hutchinson, G.E. 1957. Concluding remarks. Cold Springs Harbor Symposia on Quantitative Biology 22: 415427.

Levins, R. 1968. Evolution in Changing Environments. Princeton University Press, Princeton, New Jersey. 120 p.

Little, T.M. y F.J. Hills. 1991. Métodos Estadísticos para la Investigación Agraria. Editorial Trillas, México, México. $270 \mathrm{p}$.

Scherer, G. 1983. Diagnostic key for the Neotropical alticine genera (Coleoptera: Chrysomelidae: Alticinae). Entomologische Arbeiten aus dem Museum G. Frey 31/32: 1-89.

Vecco G., C.D. 2006. Aportes para el desarrollo de una metodología de muestreo de artrópodos en el cultivo de sacha inchik (Plukenetia volubilis L.) en la selva peruana. p. 110 En: Sociedad Entomológica del Perú. 2006. Convención Nacional de Entomología, Programa y Resúmenes. Lima, Perú.

Recibido para publicación el 9 de abril de 2010 